\title{
The efficacy of repetitions-in-reserve vs. traditional percentage-based resistance training: a 4-week pre-season randomized intervention in elite rugby league players
}

\author{
Jonathan Sinclair $^{1}\left[\right.$ C $\cdot$ Christopher James Edmundson ${ }^{1} \cdot$ Ian Bentley $^{1,2}$
}

Received: 22 February 2021 / Accepted: 7 September 2021 / Published online: 16 September 2021

(c) The Author(s) 2021

\begin{abstract}
Purpose This two-experiment study aimed to examine (1) the accuracy and reliability of repetitions-in-reserve and (2) using a randomized trial, the efficacy of repetition-in-reserve compared to traditional percentage-based training during a 4-week pre-season strength training block in elite rugby league players.

Methods In experiment 1, participants performed deadlift, bench press and weighted pull up exercises for 3, 6, and 9 repetitions to failure using self-selected loads. This was undertaken on two occasions, separated by 8 -weeks. In experiment 2 , participants were randomly separated into either repetitions-in-reserve or percentage-based training groups. They completed a 4-week pre-season training block with training prescribed based on the group to which they were assigned. Measures of body mass and 3 repetition max strength in the aforementioned exercises were measured pre and post intervention.

Results Experiment 1 showed that repetitions-in-reserve exhibited generally acceptable levels of accuracy and moderate-good levels of reliability. However, the deadlift when the required number of repetitions was 6 and 9 and the bench press when number of repetitions was 9 were not associated with acceptable levels of accuracy and reliability, respectively. Experiment 2 showed that there were no differences between repetitions-in-reserve or percentage-based training group for the strength outcomes, although there were significant increases in body mass in the percentage-based group (pre $=85.6 \pm 10.6 \mathrm{~kg}$, post $=86.4 \pm 10.8 \mathrm{~kg}$ ).

Conclusion Repetitions-in-reserve is generally associated with acceptable levels of accuracy and moderate-good levels of reliability, although there were some exceptions for the deadlift in relation to accuracy and bench press for reliability. Experiment 2 shows that although there were no differences between groups for the strength-based outcomes, significant increases in body mass in the percentage-based group may make this approach a more effective approach to prepare players for the rigors of the rugby league season.
\end{abstract}

Keywords Strength and conditioning $\cdot$ Autoregulation $\cdot$ Resistance training $\cdot$ Rugby league $\cdot$ Repetitions-in-reserve

\section{Introduction}

Rugby league is an intermittent collision sport, characterized by bouts of high intensity running and tackling [1]. Rugby league necessitates high aerobic and anaerobic fitness in

Jonathan Sinclair

jksinclair@uclan.ac.uk

$1 \quad$ Research Centre for Applied Sport, Physical Activity and Performance, Faculty of Allied Health and Wellbeing, School of Sport and Health Sciences, University of Central Lancashire, Preston PR1 2HE, Lancashire, UK

2 Wigan Warriors RLFC, Wigan, Lancashire, UK addition to speed and muscular strength/power to compete at elite level [2]. The importance of strength and power in elite rugby league is clear; with stronger and more powerful players more likely to access higher levels of competition [3], strong associations between upper body strength and tackling ability [4], and increases in strength being associated with significant reductions in post-game fatigue indicated through reduced blood creatine kinase levels [5].

As rugby league athletes play regularly during the season, they have limited time to prepare for the demanding aspects of the sport between games [6]. Pre-season represents a short and intense training phase prior to the beginning of the season that provides strength and conditioning practitioners with a short window of opportunity to significantly 
enhance aspects of physical conditioning including strength and hypertrophy $[7,8]$. Once competition resumes, resistance training is dramatically reduced, whilst the volume of game specific strategic and skills-based training is increased. As improvements in strength, power and hypertrophy important to rugby league performance are limited by the brevity of pre-season training blocks [9], it is imperative that resistance training programming during this phase is as effective as possible.

The implementation of a periodized approach whereby load, volume and frequency are manipulated, is essential to maximize improvements in hypertrophy, strength, and power [10]. The choice of load has habitually been determined as a percentage of the athlete's one-repetition maximum (1RM), a modality known as percentage-based training [11]. However, using a percentage-based approach to prescribe training load may not account for acute variations in training performance that occur during intense pre-season physical conditioning regimes, and may be impractical in programs with multiple resistance exercises due to the requirement to regularly undertake maximal strength assessments to prescribe training loads [12].

An alternative to percentage-based training is autoregulation, which is characterized by the self-management of both load and volume, based on the individual athlete's acute performance on the training day to meet sessional goals [13]. The principles of autoregulation are centered around the ability to allow instantaneous adjustments of load or volume within a training session to ensure that the appropriate level of training stimulus is experienced [14]. Of the existing autoregulation modalities, repetitions-in reserve, whereby athletes self-estimate the number of repetitions that they are able to produce during a specific set before experiencing task failure has become popular in recent years [11]. Repetitionsin-reserve represents a potentially attractive approach, as it delivers a subjective mechanism by which training can be autoregulated which can be accomplished without further specialized equipment [14].

Peer reviewed research shows that trained athletes accustomed to a given exercise are able to accurately self-select a load that will result in task failure within a prescribed/ desired number of repetitions [15-17]. Hughes et al. [16] examined repetitions-in-reserve reliability during squat, bench press, prone row, and overhead press exercises using both free weights and smith machines at 65,75 , and $85 \%$ 1RM. The above was repeated with 4-7 days apart and participants verbalized when they could only perform 2 more repetitions whilst performing the set to failure. Based on intraclass correlation (ICC) thresholds [18] reliability was moderate-good throughout ( $\mathrm{ICC}=0.59-0.83$ ) (except the squat exercise ICC $=0.42-0.49$ ) and no systematic differences in reliability between exercises or loads were shown. However, reliability was consistently greater when using free weights $(\mathrm{ICC}=0.49-0.83)$ compared to the smith machine (ICC $=0.42-0.78)$. Importantly however, there is currently no published research examining the accuracy and reliability of repetitions-in-reserve based prescription of resistance training in elite rugby league. Similarly, there is also no research concerning the efficacy of repetitions-in-reserve based training compared to traditional percentage-based approaches during pre-season training in elite rugby league players.

Therefore, the aim of the current study through a twoexperiment approach was (1) to examine the accuracy and reliability of repetitions-in-reserve in elite rugby league players and (2) to examine using a randomized trial the efficacy of a repetitions-in-reserve based training compared to a traditional percentage-based approach in terms of mediating strength related improvements during a 4-week period of pre-season training in elite rugby league players. A study of this nature may inform both strength and conditioning coaches and rugby league athletes of the most effective approach for the prescription of resistance training.

\section{Methods}

\section{Ethical approval}

For each investigation, participants provided written informed consent and ethical approval (REF: STEMH 1045) was obtained from the University of Central Lancashire, in accordance with the principles documented in the Declaration of Helsinki.

\section{Experiment 1}

\section{Participants}

Twenty-three male professional rugby league players (mean \pm standard deviation: age: $18.2 \pm 0.9$ years: body mass: $86.9 \pm 8.7 \mathrm{~kg}$ : stature: $181.8 \pm 6.3 \mathrm{~cm}$ and BMI: $26.3 \pm 1.7 \mathrm{~kg} / \mathrm{m}^{2}$ ) contracted to a Super League club in the United Kingdom volunteered to take part in this experiment. All participants were professional players from a Super League squad and had at least 3 years of resistance-based strength and power training experience.

\section{Procedure}

All testing was completed over two separate sessions with 8 weeks separating them. In each session, participants went through a standardized ramp-based warm up protocol, including a pulse raiser, dynamic stretches and potentiation exercises. After the standardized warm up protocol, one of three resistance-based exercises were randomly selected; 
either deadlift, bench press or weighted pull up. Following this, on the specific exercise that was randomly selected first, participants self-selected progressive resistance-based repetitions starting with $8-10$ repetitions on a light load, then 6-8 repetitions on a moderate load finishing with 2-3 repetitions on a heavy load. After a recovery period of $4 \mathrm{~min}$, participants then self-selected the three loads with which they believed they could perform 3, 6, and 9 repetitions to failure on the aforementioned exercise. Using these three self-selected loads, participants completed each one of them to failure across three separate sets (undertaken in a randomized order), with 4 min of recovery enforced between sets. The participants were under the supervision of an accredited strength and conditioning coach (United Kingdom Strength and Conditioning Association-UKSCA) throughout the data collection procedure. They were instructed to maintain an equal concentric and eccentric duration for each repetition with no pause between these phases or between each repetition. The actual number of repetitions performed at each load was recorded. Participants then completed the same testing protocol on the other two exercises.

After the first exercise was undertaken using the methodology described above, the same process was repeated with the remaining two exercises. Between each exercise, a recovery period of 6 min was provided. Throughout the testing process failure was determined when participants could not move the bar or themselves (during the pull-up) concentrically or they were not able to complete a repetition with the correct range of motion. After a period of 8 weeks (as outlined above) the same process was repeated in session number 2 .

\section{Statistical analyses}

Accuracy between the criterion and actual number of performed repetitions was examined firstly using the standard error of measurement (SEM) and it's 95\% confidence intervals (CI) in accordance with Steele et al. [17]. Furthermore, one-sample $t$-tests were used to assess whether significant differences existed between the criterion values of 3, 6 and 9 and the actual number of repetitions completed. In accordance with Hughes et al. [16], the 95\% CI's from the one-sample $t$-tests were examined to ensure that they did not exceed 1 repetition. This value was selected as the smallest measurable difference between criterion and actual performed repetitions, as in practice differences cannot be a portion of a repetition. Cases with no significant differences between criterion and actual repetitions and with 95\% CI's $<1$ repetition, were deemed to be accurate [16]. Finally, to examine differences in the number of repetitions performed between the three exercises for the participants 3, 6 and 9-repetitions-in-reserve estimates, repeated measures linear mixed models were used, with exercise condition modelled as a fixed factor and random intercepts by participants included [19].

In addition, the reliability of the number of repetitions performed between the two sessions was examined firstly using SEM's and their 95\% CI's. Furthermore, repetitions between the two sessions were further examined using ICC's ${ }_{(3,1)}$, which were interpreted as per previously established guidelines $<0.5=$ poor, $0.5-0.75=$ moderate, $0.75-0.9=$ good and $>0.90=$ excellent [18]. To determine whether there were significant differences between the number of repetitions performed in each session repeated measures linear mixed models were used, with time (i.e. session 1 and session 2) modelled as a fixed factor and random intercepts by participants included [19]. All analyses for statistical significance were conducted using SPSS v27 (IBM, SPSS). For linear mixed models the mean difference (b), $t$-value and $95 \%$ confidence intervals of the difference are presented and statistical significance for all analyses is accepted as the $p<0.05$ level.

\section{Experiment 2}

\section{Participants}

The same participants from experiment 1 took part in experiment 2. Participants were withdrawn from the study if they missed more than 3 training sessions during the testing or intervention periods. Two participants thus had to be removed from the study leaving twenty-one participants in total. Participants were allocated to either the repetitionsin-reserve or percentage-based group using a computer program (Random Allocation Software).

\section{Procedure}

Both training intervention groups were incorporated into the player's traditional preseason programme. The player's pre-season training program is split into 4-week blocks by their coaches, designed to focus on distinct components of rugby league fitness. This enabled all participants to be exposed to the same standardised training programme in the 4-weeks immediately prior to this study. This 'prior' programme had a strength-endurance emphasis which was designed to prepare the players for the subsequent 'strength' phase of pre-season training examined as part of the 4-week intervention phase monitored across both training groups in this experiment. The interventions were scheduled over a 4-week period, which involved $3 \times 45$ min resistance-based gym sessions and $4 \times 70$ min technical field-based sessions per week (Table 1).

In the percent-based training group, for all exercises including the three experimental lifts, participants started week 1 at $65 \%$ of their one-repetition maximum undertaking 
Table 1 Weekly pre-season training details for the players

\begin{tabular}{|c|c|c|c|c|c|c|c|}
\hline & Monday & Tuesday & Wednesday & Thursday & Friday & Saturday & Sunday \\
\hline $\mathrm{am}$ & & & Off & & Off & Technical session & Off \\
\hline $\mathrm{pm}$ & $\begin{array}{l}\text { Gym + Tech- } \\
\text { nical session }\end{array}$ & $\begin{array}{l}\text { Gym }+ \\
\text { Technical } \\
\text { session }\end{array}$ & & $\begin{array}{l}\text { Gym }+ \\
\text { Technical } \\
\text { session }\end{array}$ & & & \\
\hline
\end{tabular}

8-10 repetitions split across $3-4$ sets. In week 2 the loads for all exercises were increased to $80 \%$ of one repetition maximum and repetitions were correspondingly reduced to between 5 and 8 across the same number of sets as week 1 . In week 3 loads for each exercise were increased to $90 \%$ of one repetition maximum and repetitions were correspondingly reduced to between 3 and 6 across the 3 sets. In week 4 loads were further enhanced to $95 \%$ of one repetition maximum and repetitions were correspondingly altered to between 3 and 8 across 2 sets (Table 2a). In the repetitionsin-reserve training group, participants started week 1 undertaking 8-10 repetitions across 3-4 sets for each exercise, using a self-selected load for which they could perform the desired number of repetitions whilst leaving 6 repetitions-inreserve. In week 2 the participants undertook 5-8 repetitions across 3-4 sets for each exercise, using a load for which they could perform the desired number of repetitions whilst leaving 4 repetitions-in-reserve. In week 3, participants undertook 3-6 repetitions across 3 sets for each exercise, using load that they could perform the desired number of repetitions whilst leaving 2 repetitions-in-reserve. In week 4 , participants undertook 3-8 repetitions across 2 sets for each exercise, with a load that they could perform the desired number of repetitions whilst leaving 1 repetition in reserve [14] (Table 2b). The total training volume undertaken by each training group during the 4-week intervention period was recorded.

\section{Testing protocols}

Identical testing protocols were implemented at baseline and following the 4-week intervention. Participants completed a battery of testing to provide quantitative information required to examine the efficacy of both training groups. The players' 3 repetition maximum (3RM), for the deadlift, bench press and weighted pull-up exercises were carried out on the same day for both groups during a single gym session, with the bench press, deadlifts and pull ups being measured on Monday, Tuesday and Thursday, respectively. The 3RM values were then used as indices of strength for these exercises at baseline and post-intervention. Each testing week (i.e. at baseline and after the 4-week intervention) commenced on a Monday, following a period of $24 \mathrm{~h}$ rest as players do not train on Sunday (Table 1). Participants were instructed not to consume any alcohol during this period and continue with their typical training day diet. All participants were familiar with 3 repetition maximum testing protocols and both body mass and stature were recorded during the Monday testing session.

\section{Statistical analyses}

Comparisons between participant characteristics at baseline between the two groups were undertaken using linear mixed models, with group modelled as a fixed factor and random intercepts by participants [19]. In addition, differences between the two groups in total training volume during the 4-week intervention in the three experimental exercises were examined using linear mixed models with group modelled as a fixed factor and random intercepts by participants [19]. To examine whether there was a main effect of time across both groups, mediated by the 4-week intervention, repeated measures linear mixed models were used with time (i.e. pre-post) modelled as a fixed factor and random intercepts by participants [19]. Furthermore, to determine whether there were differences between the two training groups, after the 4-week intervention, linear mixed models with group modelled as a fixed factor and random intercepts by participants were adopted adjusted for baseline values modelled as continuous fixed covariates [20]. All analyses for statistical significance were conducted using SPSS v27 (IBM, SPSS). For linear mixed models the mean difference $(b), t$-value and $95 \%$ confidence intervals of the difference are presented and statistical significance for all analyses is accepted as the $p<0.05$ level.

\section{Results}

\section{Experiment 1}

\section{Accuracy}

Figure 1 shows the actual number of repetitions performed for the three exercises in each criterion repetition condition and the statistical analyses are presented in Table 3. Overall, with the exception of the bench press when the criterion number of repetitions was 9 , participants were able to perform more repetitions than criterion. Importantly, generally acceptable levels of accuracy were observed, with the 
Table 2 Training program information for each group

\begin{tabular}{|c|c|c|c|c|c|c|c|c|}
\hline \multirow{3}{*}{ axercise } & \multicolumn{8}{|c|}{ Percent based training } \\
\hline & \multicolumn{2}{|c|}{ Week $1-65 \%$} & \multicolumn{2}{|c|}{ Week 2-80\% } & \multicolumn{2}{|c|}{ Week 3-90\% } & \multicolumn{2}{|c|}{ Week 4-95\% } \\
\hline & Sets & Reps & Sets & Reps & Sets & Reps & Sets & Reps \\
\hline \multicolumn{9}{|l|}{ Session 1} \\
\hline 1. Deadlift & 4 & 10 & 4 & 5 & 3 & 3 & 2 & 3 \\
\hline 2. Bench Press & 4 & 10 & 4 & 5 & 3 & 3 & 2 & 3 \\
\hline 3.a. Split Squats & 3 & 8 & 3 & 6 & 3 & 4 & 2 & 6 \\
\hline 3.b. Lateral Lunges & 3 & 8 & 3 & 6 & 3 & 4 & 2 & 6 \\
\hline 4.a. Bench Rows & 3 & 10 & 3 & 8 & 3 & 6 & 2 & 8 \\
\hline 4.b. Strict Press & 3 & 10 & 3 & 8 & 3 & 6 & 2 & 8 \\
\hline \multicolumn{9}{|l|}{ Session 2} \\
\hline 1. Pull Ups & 4 & 10 & 4 & 5 & 3 & 3 & 2 & 3 \\
\hline 2. Barbell Bridges & 3 & 10 & 3 & 8 & 3 & 6 & 2 & 8 \\
\hline 3.a. Back Squats & 3 & 10 & 3 & 8 & 3 & 6 & 2 & 8 \\
\hline 3.b. RDLs & 3 & 10 & 3 & 8 & 3 & 6 & 2 & 8 \\
\hline 4.a. Strict Press & 3 & 10 & 3 & 8 & 3 & 6 & 2 & 8 \\
\hline 4.b. Bench Rows & 3 & 10 & 3 & 8 & 3 & 6 & 2 & 8 \\
\hline \multicolumn{9}{|l|}{ Session 3} \\
\hline 1. Deadlift & 4 & 10 & 4 & 5 & 3 & 3 & 2 & 3 \\
\hline 2. Bench Press & 4 & 10 & 4 & 5 & 3 & 3 & 2 & 3 \\
\hline 3. Pull Ups & 4 & 10 & 4 & 5 & 3 & 3 & 2 & 3 \\
\hline 4.a. Split Squats & 3 & 8 & 3 & 6 & 3 & 4 & 2 & 6 \\
\hline 4.b. Lateral Lunges & 3 & 8 & 3 & 6 & 3 & 4 & 2 & 6 \\
\hline \multirow[t]{2}{*}{$\mathrm{b}$} & \multicolumn{8}{|c|}{ Repetitions-in-reserve } \\
\hline & \multicolumn{2}{|c|}{ Week 1-RIR 6} & \multicolumn{2}{|c|}{ Week 2-RIR 4} & \multicolumn{2}{|c|}{ Week 3-RIR 2} & \multicolumn{2}{|c|}{ Week 4-RIR 1} \\
\hline Exercise & Sets & Reps & Sets & Reps & Sets & Reps & Sets & Reps \\
\hline \multicolumn{9}{|l|}{ Session 1} \\
\hline 1. Deadlift & 4 & 10 & 4 & 5 & 3 & 3 & 2 & 3 \\
\hline 2. Bench Press & 4 & 10 & 4 & 5 & 3 & 3 & 2 & 3 \\
\hline 3.a. Split Squats & 3 & 8 & 3 & 6 & 3 & 4 & 2 & 6 \\
\hline 3.b. Lateral Lunges & 3 & 8 & 3 & 6 & 3 & 4 & 2 & 6 \\
\hline 4.a. Bench Rows & 3 & 10 & 3 & 8 & 3 & 6 & 2 & 8 \\
\hline 4.b. Strict Press & 3 & 10 & 3 & 8 & 3 & 6 & 2 & 8 \\
\hline \multicolumn{9}{|l|}{ Session 2} \\
\hline 1. Pull Ups & 4 & 10 & 4 & 5 & 3 & 3 & 2 & 3 \\
\hline 2. Barbell Bridges & 3 & 10 & 3 & 8 & 3 & 6 & 2 & 8 \\
\hline 3.a. Back Squats & 3 & 10 & 3 & 8 & 3 & 6 & 2 & 8 \\
\hline 3.b. RDLs & 3 & 10 & 3 & 8 & 3 & 6 & 2 & 8 \\
\hline 4.a. Strict Press & 3 & 10 & 3 & 8 & 3 & 6 & 2 & 8 \\
\hline 4.b. Bench Rows & 3 & 10 & 3 & 8 & 3 & 6 & 2 & 8 \\
\hline \multicolumn{9}{|l|}{ Session 3} \\
\hline 1. Deadlift & 4 & 10 & 4 & 5 & 3 & 3 & 2 & 3 \\
\hline 2. Bench Press & 4 & 10 & 4 & 5 & 3 & 3 & 2 & 3 \\
\hline 3. Pull Ups & 4 & 10 & 4 & 5 & 3 & 3 & 2 & 3 \\
\hline 4.a. Split Squats & 3 & 8 & 3 & 6 & 3 & 4 & 2 & 6 \\
\hline 4.b. Lateral Lunges & 3 & 8 & 3 & 6 & 3 & 4 & 2 & 6 \\
\hline
\end{tabular}

Experimental exercises in bold/italic text

$R I R$ repetitions in reserve 
5.0

a

10.0

b
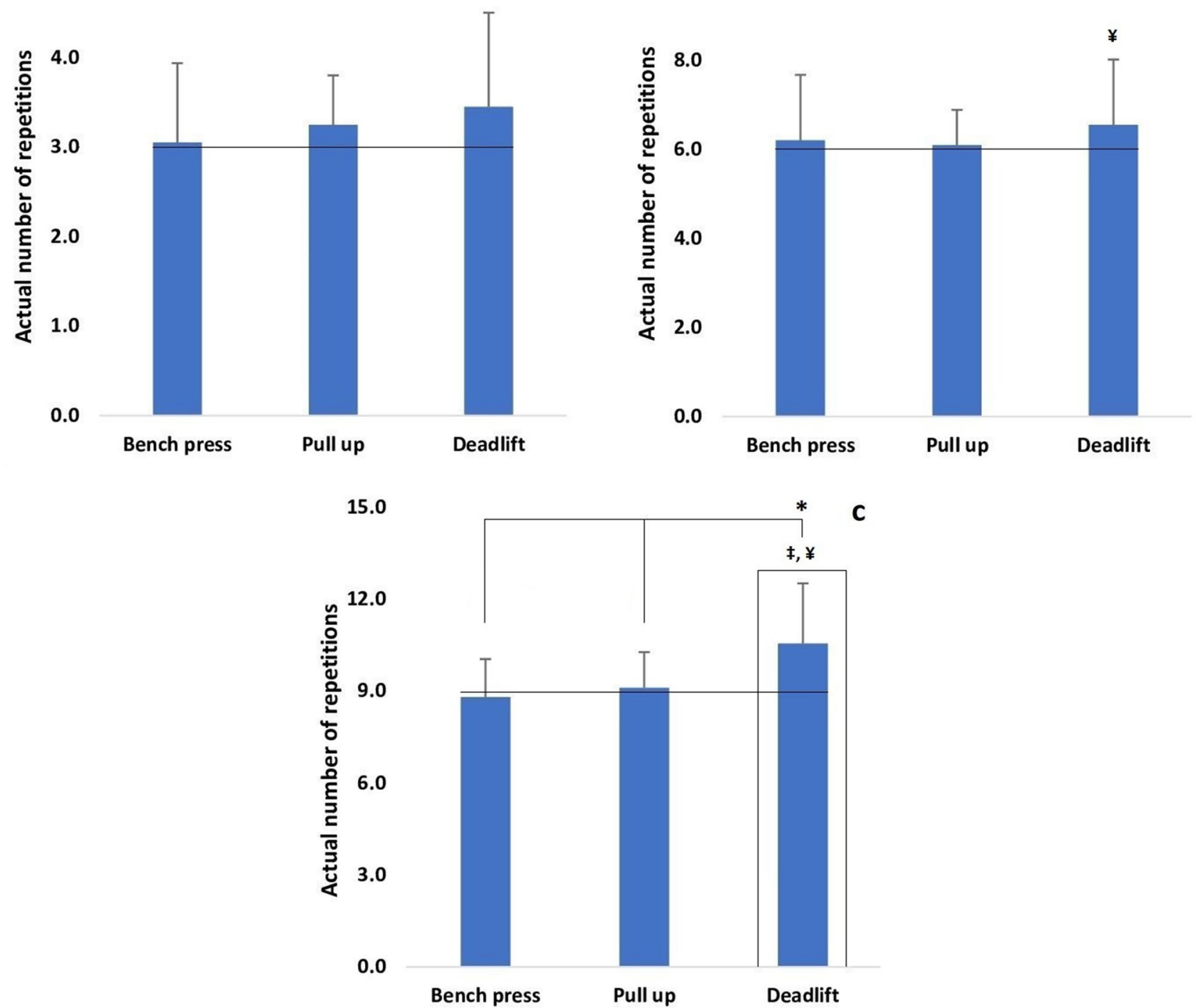

Fig. 1 Actual number of repetitions performed in each exercise in the a 3 repetitions criterion, b 6 repetitions criterion and $\mathbf{c} 9$ repetitions criterion ( $¥=$ significantly different from criterion, $¥=95 \%$ CI’s $>1$ and $*=$ significantly different from bench press and pull up exercises-horizontal lines denote the criterion number of repetitions)
Table 3 Accuracy indices for each exercise and criterion repetition condition

\begin{tabular}{|c|c|c|c|c|c|c|}
\hline & \multirow{2}{*}{$\begin{array}{l}\text { Criterion } \\
\text { repetitions }\end{array}$} & \multirow[t]{2}{*}{ SEM repetitions $(95 \% \mathrm{CI})$} & \multicolumn{3}{|c|}{ One-sample $t$-test } & \\
\hline & & & $t$ & $p$ & $95 \% \mathrm{CI}$ & \\
\hline \multirow[t]{3}{*}{ Bench press } & 3 & $0.56(0.42-0.83)$ & 0.25 & 0.80 & $-0.37-0.47$ & \\
\hline & 6 & $1.08(0.81-1.62)$ & 0.61 & 0.55 & $-0.49-0.89$ & \\
\hline & 9 & $0.92(0.69-1.37)$ & 0.72 & 0.48 & $-0.78-0.38$ & \\
\hline \multirow[t]{3}{*}{ Pull up } & 3 & $0.41(0.30-0.61)$ & 2.03 & 0.06 & $-0.01-0.51$ & \\
\hline & 6 & $0.59(0.44-0.88)$ & 0.57 & 0.58 & $-0.27-0.47$ & \\
\hline & 9 & $0.86(0.64-1.28)$ & 0.38 & 0.71 & $-0.45-0.65$ & \\
\hline \multirow[t]{3}{*}{ Deadlift } & 3 & $0.74(0.55-1.11)$ & 1.92 & 0.07 & $-0.04-0.94$ & \\
\hline & 6 & $1.06(0.80-1.60)$ & 1.68 & 0.11 & $-0.14-1.24$ & $¥$ \\
\hline & 9 & $1.46(1.10-2.19)$ & 3.54 & 0.002 & $0.63-2.47$ & $\ddagger$ \\
\hline
\end{tabular}

$\ddagger=$ significantly different from criterion and $¥=95 \%$ CI’s $>1$ 
Table 4 Reliability indices for each exercise and criterion repetition condition

\begin{tabular}{lllllll}
\hline & $\begin{array}{l}\text { Criterion } \\
\text { repetitions }\end{array}$ & \multicolumn{2}{l}{ Performed repetitions } & SEM repetitions (95\% CI) & ICC (95\% CI) & $\begin{array}{l}\text { Significant difference } \\
\text { between session 1 } \\
\text { and 2 }\end{array}$ \\
\cline { 3 - 5 } Bench press & 3 & Session 1 & Session 2 & & $0.51(0.14-0.77)$ \\
& 6 & $2.52 \pm 1.27$ & $3.05 \pm 0.89$ & $1.12(0.84-1.68)$ & $0.67(0.36-0.85)$ \\
Pull up & 9 & $6.57 \pm 1.70$ & $6.20 \pm 1.47$ & $1.61(1.20-2.41)$ & $0.42(0.01-0.71)$ & $*$ \\
& 3 & $11.17 \pm 1.92$ & $8.80 \pm 1.24$ & $1.63(1.22-2.44)$ & $0.75(0.49-0.89)$ \\
Deadlift & 6 & $3.09 \pm 0.67$ & $3.25 \pm 0.55$ & $0.33(0.24-0.49)$ & $0.52(0.14-0.77)$ \\
& 9 & $6.43 \pm 1.31$ & $6.10 \pm 0.79$ & $1.06(0.80-1.59)$ & $0.62(0.28-0.82)$ \\
& 3 & $9.52 \pm 1.47$ & $9.10 \pm 1.17$ & $0.97(0.73-1.45)$ & $0.54(0.16-0.78)$ \\
& 6 & $3.23 \pm 1.45$ & $3.45 \pm 1.05$ & $0.76(0.57-1.16)$ & $0.50(0.11-0.76)$ \\
\hline
\end{tabular}

$*=$ significant difference between session 1 and 2

exception of the deadlift exercise when the criterion repetitions were 6 and 9 (Table 3, Fig. 1b, c). Linear mixed models also showed when the target number of repetitions was 9 , that the actual number of repetitions performed in the deadlift was significantly more than in the bench press $[b=1.75(95 \% \mathrm{CI}=0.72-2.77), t=3.46, p=0.001]$ and pull up $[b=1.45(95 \% \mathrm{CI}=0.44-2.46), t=2.92, p=0.006]$ exercises (Fig. 1c).

\section{Reliability}

Table 4 shows actual number of repetitions performed in session 1 and session 2 as well as the associated test-retest reliability indices. Overall, moderate-good levels of reliability were shown, with the exception of the bench press exercise when the criterion repetitions were 9 (Table 4) and there were no clear trends in the reliability results regarding the exercise examined or criterion number of repetitions used. In the bench press exercise, when the target number of repetitions was 9 , the number of repetitions actually performed differed significantly between session 1 and session $2[b=2.34(95 \% \mathrm{CI}=1.27-3.41), t=4.57, p=0.0002]$ (Table 4).

\section{Experiment 2}

\section{Baseline characteristics}

There were no significant differences between groups at baseline for age $[b=0.06,(95 \% \mathrm{CI}=-0.41-0.53), t=0.28$, $p=0.79]$, body mass [ $b=1.09,(95 \% \mathrm{CI}=-8.80-10.99)$, $t=0.23, p=0.82]$, stature $[b=0.52,(95 \% \mathrm{CI}=-5.41-6.44)$, $t=0.18, p=0.86]$ or BMI $[b=0.16,(95 \% \mathrm{CI}=-2.04-2.36)$, $t=0.15, p=0.89$ ] (Table 5).
Table 5 Participant baseline characteristics $($ mean \pm SD) from each group

\begin{tabular}{lll}
\hline & Repetitions in reserve & Percentage-based \\
\hline$N$ (completed) & 11 & 10 \\
Age $(\mathrm{y})$ & $17.8 \pm 0.5$ & $17.9 \pm 0.6$ \\
Stature $(\mathrm{cm})$ & $181.5 \pm 8.2$ & $182.0 \pm 4.9$ \\
Body mass $(\mathrm{kg})$ & $84.7 \pm 12.1$ & $85.8 \pm 10.7$ \\
BMI $\left(\mathrm{kg} / \mathrm{m}^{2}\right)$ & $25.7 \pm 2.7$ & $25.8 \pm 2.3$ \\
\hline
\end{tabular}

Table 6 Total training volume (Mean \pm SD) from experiment as a function of each training group

\begin{tabular}{llrlll}
\hline & \multicolumn{2}{l}{$\begin{array}{l}\text { Repetitions-in- } \\
\text { reserve }\end{array}$} & & & \multicolumn{2}{l}{ Percent based } \\
\cline { 2 - 3 } \cline { 6 - 7 } \cline { 6 - 7 } & Mean & SD & & Mean & SD \\
\hline Deadlift volume $(\mathrm{kg})$ & $17,763.6$ & 1389.4 & & $17,416.0$ & 1881.8 \\
Bench press volume $(\mathrm{kg})$ & $10,200.0$ & 1496.2 & & $10,175.5$ & 1662.5 \\
Pull up volume $(\mathrm{kg})$ & 932.9 & 528.6 & & 1344.3 & 661.1 \\
\hline
\end{tabular}

\section{Training volume}

There were no differences in training volume between training groups for the deadlift $[b=347.64$ (95\% $\mathrm{CI}=-1070.80-1766.07), t=0.51, p=0.62]$, bench press $[b=24.50(95 \% \mathrm{CI}=\mathrm{CI}=-1338.65-1387.65), t=0.04$, $p=0.97]$ or pull up $[b=411.30(95 \% \mathrm{CI}=-103.02-925.61)$, $t=1.66, p=0.11]$ (Table 6).

\section{Body mass}

There was no main effect of time $[b=0.08,(95 \%$ $\mathrm{CI}=-0.38-0.53), t=0.35, p=0.73]$, however adjusted for baseline values following the intervention, body 
mass in the percentage-based training group was significantly greater than repetitions-in-reserve $[b=0.93,(95 \%$ $\mathrm{CI}=0.12-1.74), t=2.40, p=0.03]$ (Table 7).

\section{Bench press}

There was a main effect of time $[b=3.60,(95 \%$ $\mathrm{CI}=2.47-4.73), t=6.64, p=0.00001]$ showing a significant increase in 3RM bench press performance in both groups, however adjusted for baseline values following the intervention, there were no significant differences in 3RM bench press performance between the percentagebased and repetitions-in-reserve training groups $[b=0.73$, (95\% CI $=-0.96-2.42), t=0.90, p=0.38]$ (Table 7).

\section{Deadlift}

There was a main effect of time $[b=8.77,(95 \%$ $\mathrm{CI}=5.44-12.10), t=5.48, p=0.00002]$ showing a significant increase in $3 \mathrm{RM}$ deadlift performance in both groups, however adjusted for baseline values following the intervention, there were no significant differences in 3RM deadlift performance between the percentage-based and repetitions-in-reserve training groups $[b=2.07,(95 \%$ $\mathrm{CI}=-4.33-8.47), t=0.67, p=0.51]$ (Table 7).

\section{Pull up}

There was a main effect of time $[b=5.06,(95 \%$ $\mathrm{CI}=4.11-6.01), t=11.05, p=0.00000003]$ showing a significant increase in 3RM pull up performance in both groups, however adjusted for baseline values following the intervention, there were no significant differences in 3RM pull up performance between the percentage-based and repetitions-in-reserve training groups $[b=0.99,(95 \%$ $\mathrm{CI}=-0.79-2.77), t=1.16, p=0.26]$ (Table 7).

\section{Discussion}

The aim of the current study was firstly to examine the accuracy and reliability of repetitions-in-reserve in elite rugby league players and also to examine using a randomized trial the efficacy of repetitions-in-reserve compared to a traditional percentage-based training in terms of mediating strength related improvements during a 4-week pre-season training block. This represents the first examination of repetitions-in-reserve accuracy/reliability and also efficacy in relation to traditional percentage-based training methods in elite rugby league players.

Taking into account the SEM and statistical observations from the one-sample $t$-tests, experiment 1 showed importantly that overall, participants were able to estimate their required number of repetitions-in-reserve with an acceptable degree of accuracy. Importantly, experiment 1 revealed that participants habitually were able to perform more repetitions than the criterion, an observation that concurs with those of Steele et al. [17]. However, experiment 1 did show in the deadlift exercise when the criterion was 6 and 9 repetitions, that the pre-defined thresholds for acceptable accuracy were not met [16]. This observation partially concurs with those shown by Hughes et al. [16], in relation to errors being higher when the required number of repetitions was greatest, although this was not the case for the bench press and pull up exercises. Overall, it can be concluded that in elite rugby league players repetitions-in-reserve can elicit a generally acceptable level of accuracy. However, practitioners should exercise caution when designing periodized programs involving the deadlift exercise with higher repetition ranges as it may mediate a less homogeneous training stimulus.

In addition, taking account once again of the SEM and statistical observations concerning reliability, in agreement with those of Hughes et al. [16] there was generally a moderate-good level of reliability in the number of repetitions performed between the two sessions. However, in the bench press exercise when the criterion was 9 repetitions, the levels of reliability were considered poor [18], such that
Table 7 Outcomes (Mean \pm SD) from experiment 2 as a function of each training group

\begin{tabular}{|c|c|c|c|c|c|c|c|c|c|c|}
\hline & \multicolumn{4}{|c|}{ Repetitions in reserve } & \multicolumn{4}{|c|}{ Percent based } & \multirow{3}{*}{$\begin{array}{l}\text { Main } \\
\text { effect of } \\
\text { time }\end{array}$} & \multirow{3}{*}{$\begin{array}{l}\text { Difference } \\
\text { between } \\
\text { groups }\end{array}$} \\
\hline & \multicolumn{2}{|l|}{ Pre } & \multicolumn{2}{|l|}{ Post } & \multicolumn{2}{|l|}{ Pre } & \multicolumn{2}{|l|}{ Post } & & \\
\hline & Mean & SD & Mean & SD & Mean & SD & Mean & SD & & \\
\hline Body mass (kg) & 84.5 & 12.9 & 84.2 & 12.7 & 85.8 & 10.6 & 86.4 & 10.8 & & $*$ \\
\hline 3RM deadlift (kg) & 159.3 & 22.0 & 166.1 & 19.3 & 145.0 & 17.0 & 155.8 & 17.4 & $*$ & \\
\hline 3RM bench press $(\mathrm{kg})$ & 80.3 & 11.8 & 84.4 & 10.9 & 82.6 & 16.2 & 85.7 & 14.2 & $*$ & \\
\hline 3RM pull up (kg) & 17.8 & 8.8 & 23.2 & 7.7 & 14.9 & 4.7 & 19.6 & 5.1 & $*$ & \\
\hline
\end{tabular}

$*=$ significant difference 
statistically significant differences in the number of repetitions performed between sessions were observed. However, in agreement with those of Hughes et al. [16], there did not appear to be any clear trends in the reliability results regarding the exercise examined or criterion number of repetitions used. From a practical standpoint further exploration of the 207 test-retest comparisons (23 participants $* 3$ exercises * 3 criterion repetitions) showed that (except in bench press exercise when the criterion was 9 repetitions where $\leq 1$ repetition $=22.2 \%, 2$ repetitions $=38.9 \%, 3$ repetitions $=11.1 \%$, 4 repetitions $=5.6 \%, 5$ repetitions $=11.1 \%$ and 6 repetitions $=11.1 \%$ ) the number of repetitions differed between the two sessions by $\leq 1$ repetition in $72.2 \%, 2$ repetitions in $20.2 \%, 3$ repetitions in $4.2 \%$ and 4 repetitions in $3.4 \%$ of cases. Therefore, it can be concluded that in this population repetitions-in-reserve is associated with a generally moderate-good level of reliability. However once again caution should be exercised for the bench press exercise with higher repetition ranges as a consistent training stimulus over time is less likely for this condition.

In relation to the observations from the randomized trial conducted in experiment 2 , there were no differences in training volume for any of the experimental exercises between the percentage based or repetitions-in-reserve training groups. Furthermore, it was revealed that significant improvements in all three strength exercises were mediated across the 4-week intervention period, irrespective of training group allocation. This observation was to be expected and concurs with those of Comfort et al. [8] in that significant strength improvements were noted during pre-season. However, in relation to the strength related outcomes, experiment 2 showed that there were no differences between the two volume prescription methods; indicating for group-based outcomes neither appears to be superior in mediating improvements in strength-based outcomes. Nonetheless, from a practical standpoint this observation does not necessarily mean that the two methods should be considered as equal to one another. Examination of Appendix 1 shows considerable variation in the responses to both intervention modalities, thus for future analyses it is important to consider the social, psychological or even physical characteristics that may influence athlete's response to different resistance training loading strategies.

Importantly however, although there were no differences shown in the strength related outcomes, the percentagebased training group was successful in mediating a significant increase in body mass. This observation partially supports those of Georgeson et al. [21] who showed that significant increases in body mass are typically during preseason in elite rugby league players. As the training volumes did not differ between the two groups and dietary patterns were not monitored, it is not within the scope of this investigation to determine the mechanism(s) responsible for this increase in body mass shown in the percentage-based group. Nonetheless this observation may be a noteworthy one, as increased lean body mass is associated with increased performance indices in elite rugby league players [22]. Importantly body mass values at the start of the season have also been shown to be associated with a lower incidence of injuries in rugby league [21, 23]. Furthermore, Georgeson et al. [21] have shown significant and linear decreases in body mass throughout the rugby league season, owing to the reductions in resistance training volume and frequency during the season itself. The purpose of the pre-season training block is to prepare the players for the rigors of the season. Therefore, taking this and the aforementioned association between lean body mass and performance/ injury prevention into account, percentage-based training may represent a more effective method of resistance training prescription during pre-season.

A limitation to the current investigation is the relatively short duration of the intervention. Although, adopted as part of the players strength focussed pre-season training block and sufficient to mediate statistical improvements across all three exercises in both groups; strength, hypertrophy and neural based adaptations to resistance training are mediated at different rates [24]. Therefore, due to the different loading strategies under examination, there is the potential that differences between groups may have materialized had a longer intervention been adopted. A further potential drawback is that only strength-based outcomes were examined during the intervention. Although, indices of strength are important to rugby league performance [3-5], they do not provide any direct information regarding the hypertrophy and neural based adaptations that are mediated by resistance training [25]. Future, interventions of this nature may seek to correspondingly examine electromyographic and muscle architecture-based adaptations to better understand the effects of different resistance training interventions.

\section{Conclusion}

This study examined repetitions-in-reserve accuracy/ reliability and also efficacy in relation to traditional percentage-based training methods in elite rugby league players. The present investigation showed that generally repetitions-in-reserve is associated with acceptable levels of accuracy and good-moderate levels of reliability. However, the deadlift exercise when the criterion repetitions were 6 and 9 and the bench press exercise when the criterion repetitions were 9 were not associated with acceptable levels of accuracy and reliability, respectively. In addition, the randomized trial showed that there were no differences between repetitions-in-reserve or percentage-based groups for any of the strength-based outcomes. However, in the 
percentage-based group there were significant increases in body mass. Therefore given the positive association between body mass/performance and also with body mass at the start of the season being linked to a reduced incidence of injury during the season itself, this may make this approach a more effective method during pre-season of
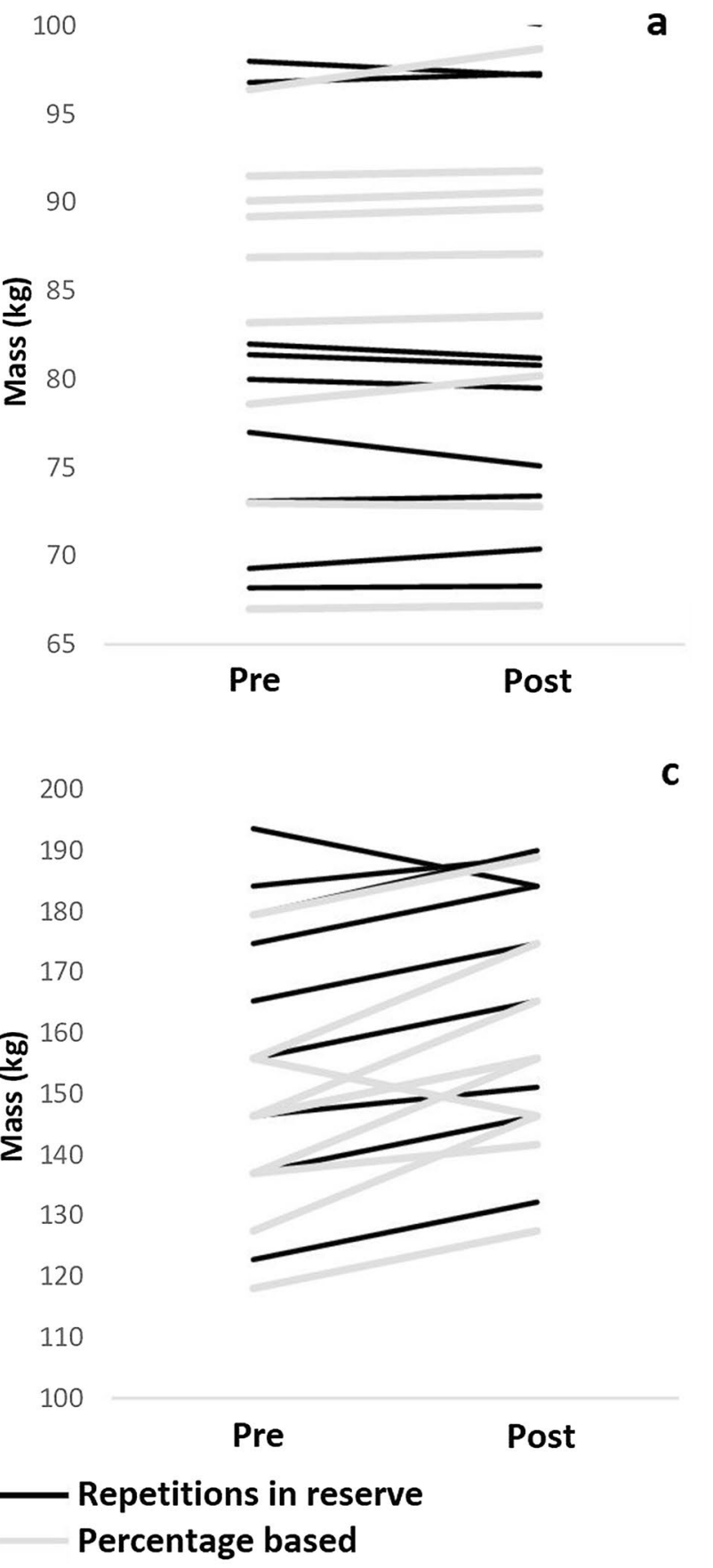

preparing rugby players for the rigors of the rugby league season.

\section{Appendix 1}

See Fig. 2.

\section{5 \\ 100 \\ 95 \\ 90

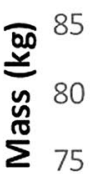 \\ 70 \\ 65 \\ 60 \\ 55}

35

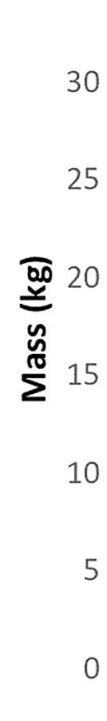

10 b
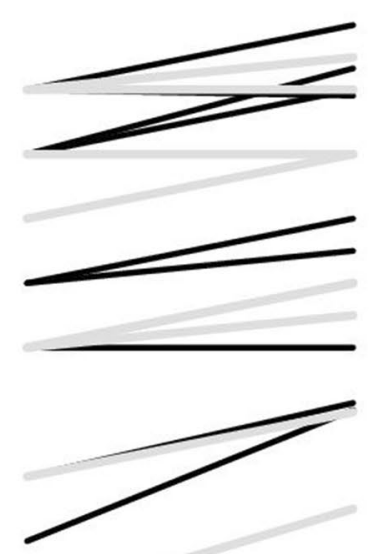

Pre

Post

d
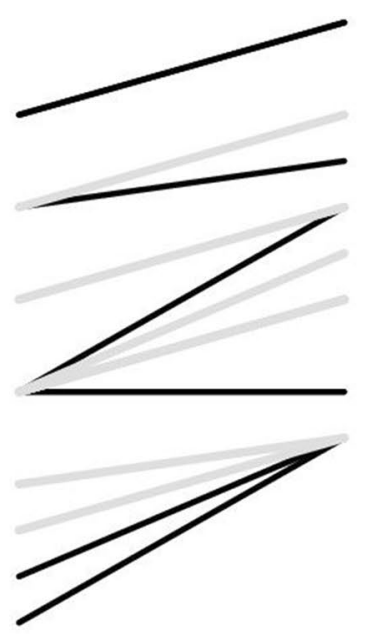

Pre

Fig. 2 Individual responses to the 4-week intervention for a body mass, $\mathbf{b}$ bench press, $\mathbf{c}$ deadlift and $\mathbf{d}$ pull up (black= repetitions-in-reserve and grey $=$ percentage based) 


\section{Declarations}

Conflict of interest The authors declare that they have no conflicts of interest.

Ethical approval Ethical approval was obtained from the University of Central Lancashire, in accordance with the principles documented in the Declaration of Helsinki.

Informed consent Participants provided written informed consent.

Open Access This article is licensed under a Creative Commons Attribution 4.0 International License, which permits use, sharing, adaptation, distribution and reproduction in any medium or format, as long as you give appropriate credit to the original author(s) and the source, provide a link to the Creative Commons licence, and indicate if changes were made. The images or other third party material in this article are included in the article's Creative Commons licence, unless indicated otherwise in a credit line to the material. If material is not included in the article's Creative Commons licence and your intended use is not permitted by statutory regulation or exceeds the permitted use, you will need to obtain permission directly from the copyright holder. To view a copy of this licence, visit http://creativecommons.org/licenses/by/4.0/.

\section{References}

1. Naughton M, Jones B, Hendricks S, King D, Murphy A, Cummins C (2020) Correction to: quantifying the collision dose in rugby league: a systematic review, meta-analysis, and critical analysis. Sports Med Open 6(1):1-26

2. Argus CK, Gill ND, Keogh JW (2012) Characterization of the differences in strength and power between different levels of competition in rugby union athletes. J Strength Cond Res 26(10):2698-2704

3. Baker DG, Newton RU (2008) Comparison of lower body strength, power, acceleration, speed, agility, and sprint momentum to describe and compare playing rank among professional rugby league players. J Strength Cond Res 22(1):153-158

4. Speranza MJ, Gabbett TJ, Johnston RD, Sheppard JM (2015) Muscular strength and power correlates of tackling ability in semi-professional rugby league players. J Strength Cond Res 29(8):2071-2078

5. Johnston RD, Gabbett TJ, Jenkins DG, Hulin BT (2015) Influence of physical qualities on post-match fatigue in rugby league players. J Sci Med Sport 18(2):209-213

6. Argus CK, Gill N, Keogh J, Hopkins WG, Beaven CM (2010) Effects of a short-term pre-season training programme on the body composition and anaerobic performance of professional rugby union players. J Sports Sci 28(6):679-686

7. Weaving D, Jones B, Marshall P, Till K, Abt G (2017) Multiple measures are needed to quantify training loads in professional rugby league. Int J Sports Med 38(10):735-740

8. Comfort P, Haigh A, Matthews MJ (2012) Are changes in maximal squat strength during preseason training reflected in changes in sprint performance in rugby league players? J Strength Cond Res 26(3):772-776

9. O'Connor DM, Crowe MJ (2007) Effects of six weeks of $\beta$-hydroxy- $\beta$-methylbutyrate (HMB) and $\mathrm{HMB} /$ creatine supplementation on strength, power and anthropometry of highly trained athletes. J Strength Cond Res 21(2):419-423

10. Lorenz D, Morrison S (2015) Current concepts in periodization of strength and conditioning for the sports physical therapist. Int J Sports Phys Ther 10(6):734-740

11. Helms ER, Cronin J, Storey A, Zourdos MC (2016) Application of the repetitions-in-reserve-based rating of perceived exertion scale for resistance training. Strength Cond J 38(4):42-49

12. Ormsbee MJ, Carzoli JP, Klemp A, Allman BR, Zourdos MC, Kim JS, Panton LB (2019) Efficacy of the repetitions-in-reservebased rating of perceived exertion for the bench press in experienced and novice benchers. J Strength Cond Res 33(2):337-345

13. Greig L, Hemingway BHS, Aspe RR, Cooper K, Comfort P, Swinton PA (2020) Autoregulation in resistance training: addressing the inconsistencies. Sports Med 50:1873-1887

14. Zourdos MC, Klemp A, Dolan C, Quiles JM, Schau KA, Jo E, Blanco R (2016) Novel resistance training-specific rating of perceived exertion scale measuring repetitions-in-reserve. J Strength Cond Res 30(1):267-275

15. Hackett DA, Johnson NA, Halaki M, Chow CM (2012) A novel scale to assess resistance-exercise effort. J Sports Sci 30(13):1405-1413

16. Hughes LJ, Peiffer JJ, Scott B (2020) Estimating repetitions-inreserve in four commonly used resistance exercises. J Strength Cond Res. https://doi.org/10.1519/JSC.0000000000003865

17. Koo TK, Li MY (2016) A guideline of selecting and reporting intraclass correlation coefficients for reliability research. J Chiropr Med 15(2):155-163

18. Steele J, Endres A, Fisher J, Gentil P, Giessing J (2017) Ability to predict repetitions to momentary failure is not perfectly accurate, though improves with resistance training experience. PeerJ 5:e4105

19. Field A (2017) Discovering statistics using IBM SPSS statistics

20. Magezi DA (2015) Linear mixed-effects models for within-participant psychology experiments: an introductory tutorial and free, graphical user interface (LMMgui). Front Psychol 6:e2

21. Georgeson EC, Weeks BK, McLellan C, Beck BR (2012) Seasonal change in bone, muscle and fat in professional rugby league players and its relationship to injury: a cohort study. BMJ Open. https://doi.org/10.1136/bmjopen-2012-001400

22. Waldron M, Worsfold P, Twist C, Lamb K (2014) Changes in anthropometry and performance, and their interrelationships, across three seasons in elite youth rugby league players. J Strength Cond Res 28(11):3128-3136

23. Gabbett TJ, Domrow N (2005) Risk factors for injury in sub-elite rugby league players. Am J Sports Med 33(3):428-434

24. Seynnes OR, de Boer M, Narici MV (2007) Early skeletal muscle hypertrophy and architectural changes in response to high-intensity resistance training. J Appl Physiol 102(1):368-373

25. Krzysztofik M, Wilk M, Wojdała G, Gołaś A (2019) Maximizing muscle hypertrophy: a systematic review of advanced resistance training techniques and methods. Int J Environ Res Public Health 16(24):1-14

Publisher's Note Springer Nature remains neutral with regard to jurisdictional claims in published maps and institutional affiliations. 